\title{
A global survey identifies novel upstream components of the Ath5 neurogenic network Marcel Souren ${ }^{* *}$, Juan Ramon Martinez-Morales ${ }^{\star * \dagger}$, Panagiota Makri*, Beate Wittbrodt ${ }^{*}$ and Joachim Wittbrodt*
} Addresses: * Developmental Biology Unit, EMBL-Heidelberg, Meyerhofstrasse, Heidelberg, 69117, Germany. ${ }^{\dagger}$ Centro Andaluz de Biología del
Desarrollo (CABD), CSIC-Universidad Pablo de Olavide, Carretera de Utrera Km1, Sevilla, 41013, Spain.

These authors contributed equally to this work.

Correspondence: Juan Ramon Martinez-Morales. Email: jrmarmor@upo.es. Joachim Wittbrodt. Email: jochen.wittbrodt@emblheidelberg.de

Published: 7 September 2009

Genome Biology 2009, 10:R92 (doi:10.1 I86/gb-2009-10-9-r92)

The electronic version of this article is the complete one and can be found online at http://genomebiology.com/2009/10/9/R92
Received: 14 April 2009

Revised: 29 July 2009

Accepted: 7 September 2009

(C) 2009 Souren et al.; licensee BioMed Central Ltd.

This is an open access article distributed under the terms of the Creative Commons Attribution License (http://creativecommons.org/licenses/by/2.0), which permits unrestricted use, distribution, and reproduction in any medium, provided the original work is properly cited.

\begin{abstract}
Background: Investigating the architecture of gene regulatory networks (GRNs) is essential to decipher the logic of developmental programs during embryogenesis. In this study we present an upstream survey approach, termed trans-regulation screen, to comprehensively identify the regulatory input converging on endogenous regulatory sequences.

Results: Our dual luciferase-based screen queries transcriptome-scale collections of cDNAs. Using this approach we study the regulation of Ath5, the central node in the GRN controlling retinal ganglion cell (RGC) specification in vertebrates. The Ath5 promoter integrates the input of upstream regulators to enable the transient activation of the gene, which is an essential step for RGC differentiation. We efficiently identified potential Ath5 regulators that were further filtered for true positives by an in situ hybridization screen. Their regulatory activity was validated in vivo by functional assays in medakafish embryos.

Conclusions: Our analysis establishes functional groups of genes controlling different regulatory phases, including the onset of Ath5 expression at cell-cycle exit and its down-regulation prior to terminal RGC differentiation. These results extent the current model of the GRN controlling retinal neurogenesis in vertebrates.
\end{abstract}

\section{Background}

Gene regulatory networks (GRNs) determine the animal body plan and cooperate to specify the different cell types of the organism. They have evolved to integrate and precisely control developmental programs. While changes in the periphery of the networks may lead to subtle changes in body plan mor- phology, the GRN core architecture around central nodes remains more conserved [1].

In the vertebrate retina, the control of retinal progenitor cell (RPC) fate-choice and differentiation depends on the synchronization of intrinsic genetic programs and extrinsic sig- 
nals. A hierarchical GRN controls the sequential generation of the different retinal cell types during embryogenesis [2]. There is increasing evidence that timing of cell cycle exit and cell-fate choice are closely linked, as cells forced to exit the cell cycle prematurely were more likely to adopt an early cell fate and vice versa [3-6]. The position of RPC nuclei within the developing neuroretina depends on the phase of the cell cycle. S-phase takes places at the basal side of the epithelium, while M-phase nuclei are located at the apical side [7-9].

In all vertebrate species analyzed, retinal ganglion cells (RGCs) are the first to be generated within an otherwise undifferentiated epithelium. The basic helix-loop-helix (bHLH) transcription factor Ath5 is the central switch in the GRN governing RGC neurogenesis. Loss of Ath 5 in mouse and zebrafish leads to a complete absence of RGCs and an increase of later born cell types, such as amacrine cells and cone photoreceptors [10-12]. Gain-of-function experiments in chicken and frog showed that Ath5 promotes RGC formation at the expense of other cell types $[13,14]$. The onset of Ath5 expression in newborn RGCs coincides with the exit from the cell cycle $[15,16]$. RGCs are specified in a neurogenic wave that spreads across the retina similar to the morphogenetic furrow that moves through the eye imaginal disc in Drosophila [17]. RGCs first appear ventro-nasally close to the optic stalk in zebrafish $[18,19]$. Subsequently, a wave of differentiating cells spreads to the periphery of the eye [20-22]. In medaka, newborn RGCs first appear in the center of the retina at the initiation stage (IS). During the progression stage (PS), neuronal differentiation proceeds towards the peripheral retina. The final stage is a 'steady wave stage' (SWS) in which newborn RGCs are found exclusively in a ring in the peripheral ciliary marginal zone. At this stage retinal progenitor cells derived from the ciliary marginal zone undergo neurogenesis and contribute to the layered structure of the central retina (Figure 1a).

The initiation of Ath5 expression and RGC differentiation depends on extra-cellular signals emanating from the optic stalk [19]. Extra-cellular signals involved in RGC formation include members of the Wnt and fibroblast growth factor (FGF) signaling cascade [23,24]. Soluble molecules produced by RGCs themselves, such as Fgf19 and Sonic hedgehog (Shh), have been implicated in the spread of the wave [25,26]. However, the Ath 5 promoter is activated in a wave-like manner even in the absence of RGCs in the zebrafish Ath 5 mutant lakritz. Mutant cells initiate Ath5 expression according to their initial position when transplanted to a different spot in the retina [27]. These data support a cell-intrinsic mechanism triggering Ath 5 expression. A small number of transcription factors have been shown to directly regulate Ath 5 expression in vivo (Figure $1 \mathrm{~b}$ ). The bHLH factor Hes1, activated downstream of the Notch pathway, has been shown to repress the formation of RGCs and other cell types in mouse, such as rod photoreceptors and horizontal and amacrine cells prior to the onset of neurogenesis $[28,29]$. In chicken, Hes1 was shown to repress Ath5 in proliferating RPCs [30]. After the onset of Ath 5 expression at the last mitosis, Ath 5 protein binds to and activates its own promoter [31,32]. Additionally, it also receives positive regulatory input from Ngn2, NeuroM and Pax6 [33-36]. The terminal differentiation of RGCs is accompanied by a downregulation of Ath5, which is no longer expressed in mature neurons [30].

The Ath 5 promoter integrates important upstream input to initiate RGC specification [2]. However, little is known about the transcriptional regulators governing the onset of Ath5 expression at the transition from proliferating progenitors to early post-mitotic cells and its downregulation prior to terminal differentiation. It is, for example, unclear how general cell cycle regulators may impinge upon the GRN controlling RGC specification.

The analysis of upstream gene regulation for key developmental genes has mainly focused on the dissection of the cisregulatory logic using approaches such as promoter bashing or computational predictions. The systematic identification of trans-acting genes regulating a defined promoter has so far relied on binding assays such as yeast-one-hybrid assays [37]. Yeast-one-hybrid assays have been used to identify proteinDNA interactions based on the activity of a DNA-binding protein fused to an activating or repressing domain. Recently, the use of bacterial hybrid-screening technology and oligo arrays have overcome some of the limitations of the extensive cloning required $[38,39]$, but these methods still depend on the generation of fusion proteins and only allow testing of a limited number of protein-DNA interactions. Initial attempts have been made to overcome these limitations by the use of luciferase-reporter based assays that employ synthetic reporter constructs [40].

Here, we present an upstream regulation survey, termed trans-regulation screen (TRS), using two nested screens to identify novel regulatory input on the Ath 5 promoter (Figure 1c). The dual luciferase-based screening strategy allows surveying transcriptome-scale collections of full-length native cDNAs. They are tested for their activating or repressing properties on an endogenous promoter in vertebrate cells. The candidates were further filtered in a semi-automated in situ hybridization screen. Through this approach we have identified novel regulators of Ath5, and gained insight into the control of the retinal neurogenic network. Here we show the power of TRS technology as an upstream approach to survey developmental regulatory networks.

\section{Results \\ The trans-regulation screen identifies candidate regulators of Ath5}

To gain insight into the molecular mechanisms controlling the dynamic expression of Ath5, we explored the regulatory logic of a medakafish 3 -kb promoter fragment that fully reca- 


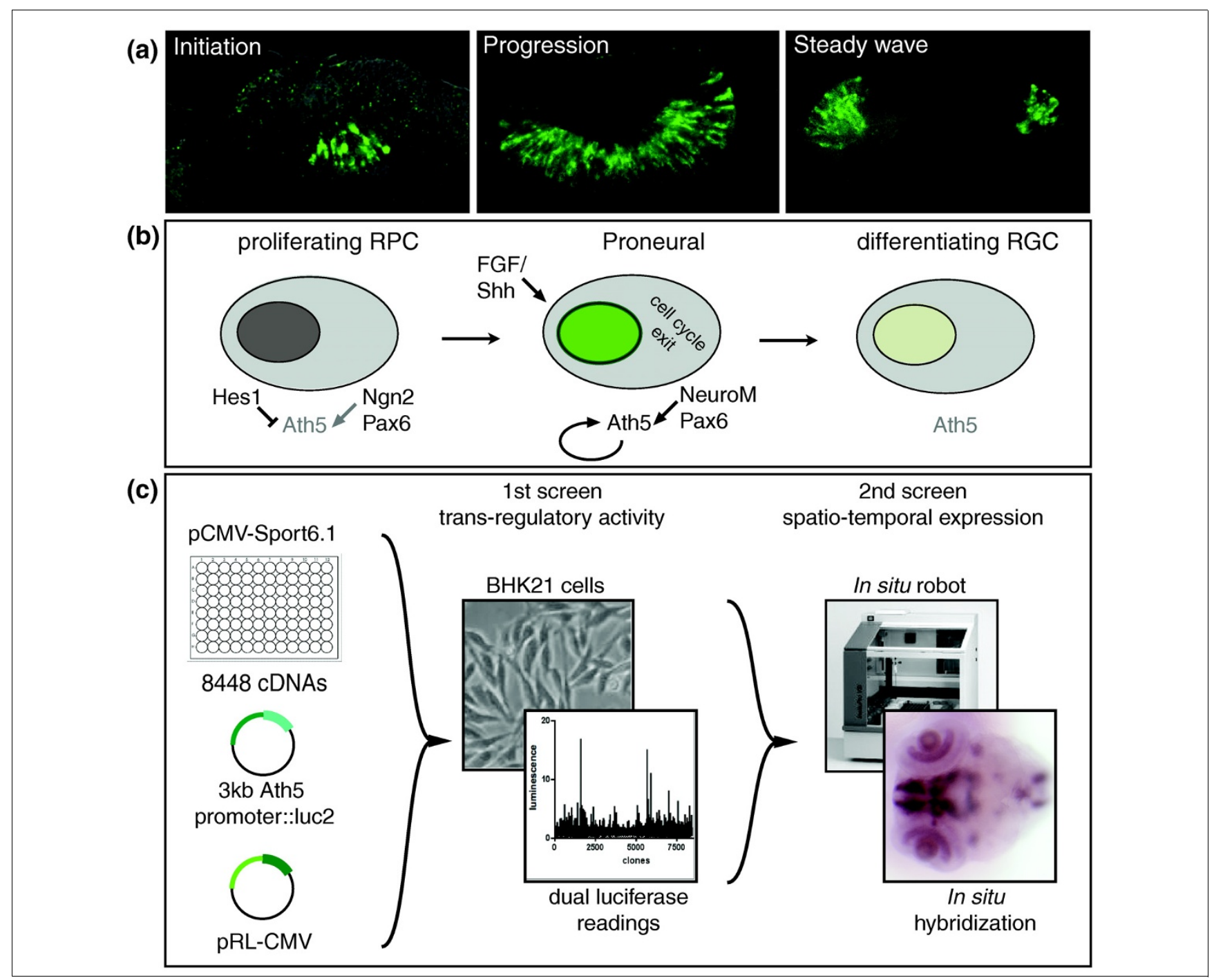

\section{Figure I}

Screen overview. (a) Neurogenic wave in medaka. Single confocal sections through eye stained for Ath5 mRNA at the level of the lens. The sections show the neurogenic wave during its initiation, progression and steady wave stage. (b) Current model of Ath5 regulation. Three stages of Ath5 regulation have been identified: initial repression in proliferating RPCs; activation and maintenance in the proneural state around the exit of cell cycle by Fgf8, NeuroD, Pax6, and Ath5 itself; and finally terminal downregulation in differentiating RGCs. (c) Schematic overview of transregulation screen. We individually cotransfected 8,448 Oryzias latipes CDNAs with pGL3 Ath5::Luc and a cytomegalovirus (CMV)-driven Renilla luciferase control vector (pRL-CMV) into BHK2I cells in 96-well plates. Each transfection was carried out in triplicate. Identified candidates were filtered using semi-automated in situ hybridization. FGF, fibroblast growth factor; Shh, Sonic hedgehog.

pitulates the endogenous Ath5 expression pattern in vivo [31]. Using this promoter, we tested the ability of individual cDNAs to either activate or repress a luciferase reporter construct upon co-expression in BHK21 cells.

We employed a sequenced and arrayed medaka cDNA expression library, comprising unigene full-length clones in pCMVSport6, to individually test 8,448 genes. Our high-throughput trans-regulation screen allows efficient and reliable normalization using a second control reporter. We co-transfected each cDNA with the Ath5 firefly luciferase reporter (Ath5::luc2) and a cytomegalovirus (CMV)-driven Renilla luciferase control vector (pRL-CMV) in triplicate in a 96-well format. Luminescence levels of reporter and control were recorded after $48 \mathrm{~h}$ (Figure 1c). As a control we tested in parallel the known regulators of Ath 5 - Hes-1, Pax6 and Ath5 itself - under screening conditions. We confirmed that Hes1 has a strong repressive activity on the 3 -kb promoter fragment, while Pax6 and Ath5 can activate the promoter in a dose-dependent manner (Figure S1 in Additional data file 1) as previously reported [34-36].

The inclusion of the CMV-driven Renilla luciferase control [41] in the screen reduced the average standard deviation 
from $35.5 \pm 80.2 \%$ to $17.3 \pm 19.3 \%$ and was essential to correct for unspecific variation such as initial cell number, cell proliferation rate and transfection efficiency. As quality thresholds, we discarded those clones for which Renilla luminescence values were below 8,000 relative luminescence units, reflecting low cell numbers and/or general toxicity of the transfected construct. In addition, clones yielding firefly luminescence values smaller than ten times the background signal (ten raw units) were discarded. All raw luminescence readings were stored in a FileMaker database. Median values were calculated and normalized and statistics were generated using Prism software (supplementary material and methods in Additional data file 1). For $87.7 \%$ of the clones all three assays were successful, reflecting the robustness and the reliability of the screening setup (Figure 2a). To remove the plate-to-plate variation, we normalized each ratio (firefly over Renilla) against the average of all ratios in the plate. This approach has been previously employed [42] and was used as all plates are likely to only contain a very small number of regulators. Figure $2 b$ represents the normalized ratios for all clones in a frequency distribution histogram in log-space.
As only a small number of cDNAs are likely to have an effect on the Ath 5 promoter, the variation of luminescence ratios around the average can be regarded as random for almost all cDNAs while values outside a normal distribution curve are unlikely to be random variations. We therefore could fit a Gaussian normal distribution to the data (Figure 2b) and selected candidate genes based on mathematical criteria. Thus, clones with a normalized ratio of less than 0.2859 or more than 2.8732 were selected as candidates. In addition, only candidates with a standard deviation within the average standard deviation of all clones $(15.7 \pm 19.1 \%)$ were chosen. Ninety-three full-length cDNAs fulfill these criteria and can be mapped onto genes in the Ensembl gene build (Table S1 in Additional data file 1). They make up 1.1\% of the total number of clones screened. Of these cDNAs, 28 are in vitro repressors, and 65 are in vitro activators. We analyzed the Gene Ontology terms associated with the candidates using the DAVID webtools (Figure 2c). Of all candidates with a GO annotation, $45.7 \%$ are localized in the nucleus and $44.3 \%$ are nucleic acid binding factors. The screening technology therefore gives a concise list of candidates that is enriched for nuclear factors involved in gene regulation.

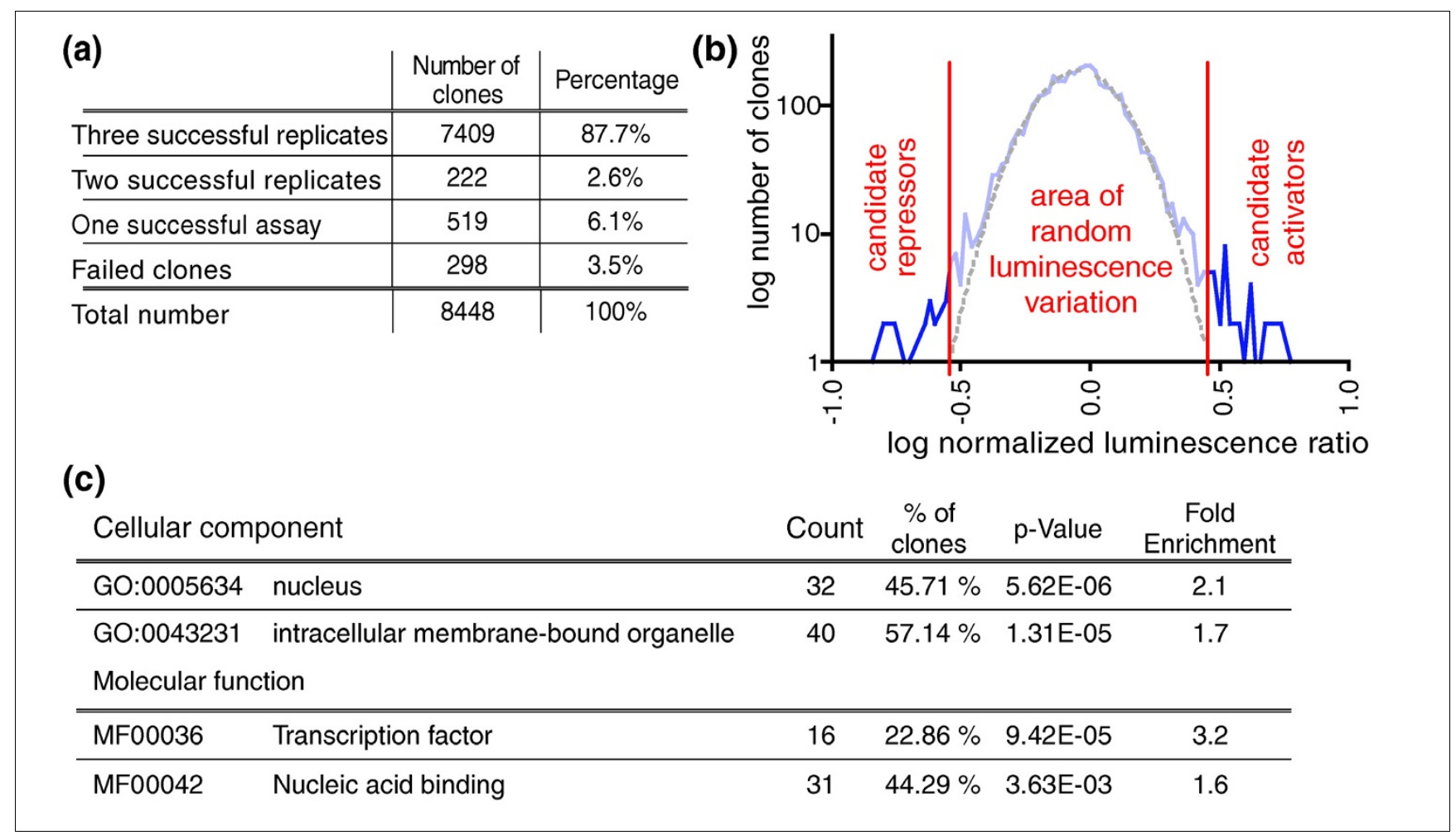

\section{Figure 2}

Screening statistics and candidate selection. (a) Screening statistics. The table lists the number of successful replicates per clone. (b) Selection of clones with non-random luminescence variation. All luminescence ratios were transformed into log-space for visualization. Luminescence ratios with a negative log value indicate a repressive effect, and positive log values an activating effect. The dotted line represents a Gaussian normal distribution fitted to the dataset. The left vertical line labels the threshold for repressors (less than $10^{-0.544}=0.2859$ ), and the right vertical line labels the threshold for activators (more than $10^{0.458}=2.8732$ ) (c) Gene Ontology analysis of candidate regulators. Candidates were analyzed for cellular localization and molecular function independently. The most abundant, non-redundant categories with a significant enrichment in the dataset compared to the genome are depicted. 


\section{Nested in situ hybridization analysis refines the dataset to $\mathbf{5 3}$ high-confidence candidates}

To assess whether the candidates can act as regulators in vivo, we determined their expression patterns. Using an in situ hybridization robot, we examined three different stages of development that coincide with the different phases of the Ath5 wave: initiation (IS, stage 24), progression (PS, stage 27) and steady wave stage (SWS, stage 31). All images of expression patterns have been submitted to the Medaka Expression Pattern Database [43] (Figure S2 in Additional data file 1). For 17 clones no expression was found at the tested stages and 23 genes were expressed in different domains of the embryo. Consistent with a function in Ath5 regulation, 10 genes were expressed ubiquitously at all time, while 43 genes were expressed specifically in the eye at one or more time points.

These specifically and dynamically expressed genes were analyzed by double fluorescence whole-mount in situ hybridization, using Ath 5 as reference probe in parallel, to determine the exact relative expression patterns of Ath 5 and the candidate regulators. According to their spatio-temporal expression, they were grouped into four categories (Table 1). Group 1 consists of 9 candidate repressors expressed in RPCs and early RGCs, group 2 of 25 candidate activators expressed in these cells. Group 3 contains three candidate activators expressed in late differentiating RGCs and group 4 contains six candidate repressors expressed in late differentiating RGCs.

The expression of group 1 genes (repressors) becomes restricted to the retinal periphery as the neurogenic wave proceeds. Genes of this group overlap with Ath 5 only in the early post-mitotic RGCs located apically in the differentiating epithelium (arrowheads in Figure $3 \mathrm{c}, \mathrm{f}$ ). They include replication complex factors MCM2 and 3 (Figure $3 a-c$; Figure $\mathrm{S}_{3} \mathrm{a}, \mathrm{b}$ in Additional data file 1), the importin-family members KPNA4 and 2 (Figure 3d-f; Figure S3c in Additional data file 1), the regulator complex protein Cnot10 and a sterol demethylase (Figure S3d-f in Additional data file 1). Representative examples of group 2 (activators) are Retinoblastoma ( $\mathrm{Rb})$, secreted frizzled related protein (sFRP) 1 and SRP40 (Figures 3g-k and 4a-c). $R b$ overlaps with Ath5 in apically located early RGCs (arrowheads in Figure 3h, i) exiting the cell cycle at all stages of the wave. $S F R P 1$ is expressed at IS and PS, but ceases to be expressed at SWS (Figure 3j, k). SRP40, a splicing factor-like protein without known function, is found in RPCs and early RGCs at SWS (Figure 4a, b).

Group 3 genes (late activators) include Islet-2 (Figure 3m, n) and Ndrg3 (Figure 4c, d). Finally, group 4 (late repressors) includes Idax, a negative regulator of the Wnt-pathway (Figure 3p, q), the nucleotide-binding protein RBPMS2 (Figure 4e, f), the zinc-finger containing protein Zfp-161 (Figure 4g, h), ELG-protein (Figure 4i, j) and the novel NHL-domain containing protein (Figure $4 \mathrm{k}, \mathrm{l}$ ). Group 3 as well as group 4 genes are co-expressed with Ath 5 only in a few terminally migrating RGCs located basally (arrowheads in Figures $3 \mathrm{r}$ and $4 f, h, j, l$ ) and maintain their expression in already localized RGCs.

These four categories define distinct regulatory activities at two critical points of Ath5 regulation, the onset of Ath5 expression in RPCs exiting the cell cycle and the sharp terminal downregulation in late migrating RGCs.

\section{Candidates that act dose-dependently are potential direct regulators of Ath5}

We further characterized the activity of individual in situ validated candidates by assessing the dose-dependence of their regulatory effect. We employed our high-throughput pipeline to perform experiments for each candidate across a wide range of concentrations. Parallel experiments using CMVand SV4O-driven reporters were performed independently as a control to exclude regulatory effects on the reference promoters. We obtained data for 45 genes expressed in the eye. Of these, 19 exhibited a clear correlation between the amount of regulator and signal strength (for the complete dataset see supplementary Table S2 in Additional data file 1). These linear dose-response relations suggest a direct regulatory activity, while non-linear relations point at a more indirect mode of activity. Consistent with a more direct regulation on Ath5, $71 \%$ of the genes annotated as nucleic acid binding showed a linear dose-response in these assays (Table S2 in Additional data file 1).

To test the direct binding of some of the regulators to the promoter, namely the bona fide transcription factors Islet1 and p65, we screened the Ath5 3-kb fragment for predicted transcription factor binding sites (TFBSs) using TRANSFAC [44]. Those TFBSs located within conserved boxes proximal to the Ath 5 transcription start site were cloned upstream of a luciferase reporter (Figure S4a in Additional data file 1). Fragments (29 bp including the TFBSs) were then assayed for their ability to mediate either Islet-1 or p65-induced transcription in a dose-response manner. Our in vitro analysis showed that selected TFBSs are functional by themselves (Figure $\mathrm{S} 4 \mathrm{~b}$ in Additional data file 1), thus suggesting that some of the identified regulators have a direct input on the Ath5 promoter.

The list of genes with a linear dose-response curve also contains enzymes, such as GPI deacetylase or thiolase, and signaling components, such as Idax and sFRP1, whose functions suggest a more upstream entry into the Ath 5 regulatory pathway. In addition, several genes with unknown function showed dose-dependent behavior in our assays. To test whether the linear dose-response of these candidates with unknown function correlates with nuclear localization, we generated carboxy-terminal green fluorescent protein (GFP)tagged proteins and analyzed their subcellular localization. Fusion constructs were co-transfected into BHK21 cells together with a red fluorescent protein membrane marker as 
Table I

List of candidates

\begin{tabular}{lll}
\hline Name & Fold-change & ID \\
\hline Group I: repressors in RPCs & & \\
AATF & $0.21 \pm 0.02$ & Rb-binding protein Che-I \\
ARGI & $0.27 \pm 0.02$ & Liver-type arginase \\
ATP-synthase & $0.28 \pm 0.04$ & ATP synthase beta chain \\
Cnot10 & $0.15 \pm 0.01$ & CCR4-NOT transcription complex, subunit 10 \\
DuS4L & $0.28 \pm 0.01$ & tRNA-dihydrouridine synthase 4 -like \\
KPNA4 & $0.25 \pm 0.04$ & Importin alpha-4 subunit \\
MCM2 & $0.27 \pm 0.00$ & DNA replication licensing factor 2 \\
USP25 & $0.28 \pm 0.05$ & Ubiquitin carboxyl-terminal hydrolase 25 \\
WDR43 & $0.29 \pm 0.04$ & WD repeat protein 43, unknown function
\end{tabular}

Group 2: activators in RPCs

Bcat2

$\mathrm{Cb} \times 7$

CEB55

GPI deacetylase

PTPN2

$\mathrm{RbI}$

SRP40

Sterol demethylase

Thiolase

TMEM79

TMP49

Transferase

Bub3

FAN

Hspl

KPNA2

MCM3

MRPL47

NHL-domain II

Ribonuclease

sFRP-I

TARBP2

Tetraspanin-9

USPI

Group 3: activators in RGCs

Ndrg3a

Islet2

Tetraspanin-3।

Group 4: repressors in RGCs

ELG protein

Idax

$\mathrm{NHL}$-protein

RBM4L

RBPMS2

Zfp 161
$2.93 \pm 0.27 \quad$ Mitochondrial branched chain aminotransferase 2

$2.96 \pm 0.14 \quad$ Polycomb group gene

$4.70 \pm 0.39$ Centrosomal protein of $55 \mathrm{kDa}$

$15.00 \pm 0.8 \mathrm{I} \quad$ Vesicular transport

$3.65 \pm 0.59 \quad$ Tyrosine-protein phosphatase non-receptor

$3.50 \pm 0.46 \quad$ Cell cycle exit, transcription factor

$2.88 \pm 0.10 \quad$ Splicing factor

$3.33 \pm 0.33$ Sterols and steroids biosynthesis, oocyte maturation

$4.98 \pm 0.32 \quad$ Trifunctional enzyme, acetyl-CoA transferase

$3.01 \pm 0.30 \quad$ Transmembrane protein, function unclear

$3.92 \pm 0.42 \quad$ Transmembrane protein, function unknown

$3.39 \pm 0.58 \quad$ Arginine n-methyl-transferase

$4.85 \pm 0.00 \quad$ Mitotic checkpoint protein

$3.28 \pm 0.28 \quad$ Associated with N-SMase activation

$11.02 \pm 1.36 \quad$ Heat shock protein I

$3.65 \pm 0.36 \quad$ Importin alpha-2 subunit,

$3.00 \pm 0.00 \quad$ DNA replication licensing factor 3

$3.33 \pm 0.00 \quad$ Mitochondrial ribosomal protein L47 isoform $b$

$3.36 \pm 0.59 \quad \mathrm{NHL}$-domain containing, unknown function

$4.15 \pm 0.09 \quad$ Ribonuclease $\mathrm{HI}$ large subunit

$2.97 \pm 0.55 \quad$ Wnt-signal regulator

$3.05 \pm 0.18$ TAR RNA-binding protein 2

$3.29 \pm 0.00 \quad$ Transmembrane protein, interacts with integrins

$3.25 \pm 0.34 \quad$ Ubiquitin carboxyl-terminal hydrolase I

$3.73 \pm 0.00 \quad \mathrm{~N}$-myc downstream regulated 3, function unknown

$5.11 \pm 0.22 \quad$ Insulin gene enhancer, transcription factor

$3.00 \pm 0.38 \quad$ Transmembrane protein, unknown function

$0.27 \pm 0.00 \quad$ mRNP complex, unknown function

$0.16 \pm 0.01 \quad$ Negative regulation of Wnt signaling

$0.27 \pm 0.00 \quad \mathrm{NHL}$-domain containing, unknown function

$0.23 \pm 0.01 \quad$ RRM-class RNA-binding protein

$0.20 \pm 0.06 \quad$ RNA-binding protein RNP-I, unknown function

$0.23 \pm 0.01 \quad$ Zinc finger, function unclear 
Table I (Continued)

List of candidates

Ubiquitously expressed regulators

$\begin{array}{lll}\text { HMG } & 2.93 \pm 0.49 & \text { HMG box DNA-binding domain } \\ \text { p65 TF } & 6.16 \pm 0.81 & \text { NF- } k B \text { transcription factor p65 } \\ \text { Beta-actin } & 0.27 \pm 0.03 & \text { Cytoskeleton } \\ \text { Tubulin alpha-IB chain } & 3.28 \pm 0.59 & \text { Cytoskeleton } \\ \text { UBR2 } & 3.18 \pm .0 .36 & \text { Ubiquitin-protein ligase E3 component N-recognin-2 } \\ \text { Uncharacterized I } & 0.22 \pm 0.01 & \text { Unknown function } \\ \text { Coiled-coil domain } & 3.25 \pm 0.43 & \text { Unknown function } \\ \text { EF-I-alpha } & 3.31 \pm 0.26 & \text { Elongation factor } \\ \text { Nfkbia } & 2.99 \pm 0.44 & \text { NF-kappaB inhibitor } \\ \text { Ankrd39 } & 5.40 \pm 0.71 & \text { Ankyrin repeat domain-containing protein 39, unknown function }\end{array}$

Candidate clones were selected based on their relative effect on the reporter construct. Out of this list, clones with a specific spatio-termporal expression in the eye were grouped into four categories (groups I to 4). An additional category contains clones expressed ubiquitously. For each clone the fold-change of reporter activity with standard deviation and a short description of the gene are shown.

a reference. The splicing factor SRP40 was used as a control for nuclear localization (Figure 4c). Our analysis showed that the zinc finger protein 161 and the ELG-protein are exclusively localized in the nucleus (Figure 4p, q). The RNA-binding protein RBPMS2 and Ndrg3 are localized in both the nucleus and cytoplasm (Figure 4n, o), suggesting that they can shuttle between these cellular compartments. In fact, nuclear localization of $\mathrm{Ndrg}_{3}$ has been recently reported in the mouse central nervous system [45]. The NHL-domain protein is excluded from the nucleus and accumulates in a perinuclear compartment, which resembles the Golgi apparatus (Figure 4r). In conclusion, the nuclear localization of four out of five uncharacterized proteins analyzed suggests that they act as direct regulators of Ath5.

\section{Clonal analysis of individual regulators in transgenic medaka embryos in vivo validates their role in $\mathbf{R G C}$ differentiation}

We complemented the characterization of candidate regulators by testing in vivo the activity of members of each of the four expression-activity categories in medaka embryos. To examine RGC differentiation, we followed the dynamic regulation of the Ath 5 promoter using a transgenic line expressing degradable GFP under the control of the Ath5 promoter (Ath5::d1GFP). Candidates were expressed in the developing neural retina in a mosaic fashion by DNA microinjection of the candidate genes under the control of the retina-specific medaka $R x 2$ promoter. Clones expressing the candidate genes were traced by co-injection of Rx2::H2A-mCherry [46]. In this mosaic situation we quantified the proportion of candidate expressing cells (red) that regulated the expression of the Ath 5 reporter (green), making the analysis independent of the total number of Ath5 positive cells. We thus determined the in vivo activity of the different candidates in the generation of Ath 5 positive cells and, hence, in RGC neurogenesis. As a baseline control, the Rx2::nuclearCherry construct was injected alone (Figure $5 \mathrm{a}$ ). In this assay the known regulators Ath 5 and Hes-1 resulted in robust activation and repression of the reporter (Figure $5 \mathrm{~b}, \mathrm{c}$ ). In agreement with the reported key role of Ath5 in RGC neurogenesis, its clonal expression was sufficient by itself to induce ectopic differentiation foci in the peripheral retina (Figure $5 \mathrm{c}$ ).

Consistent with their behavior in our transactivation screen, sFRP1, Rb1 and Ndrg3a act as activators of Ath 5 in vivo (Figure $5 \mathrm{~d}, \mathrm{f}$ ). Interestingly, although the over-expression of these activators enhanced Ath 5 expression, ectopic differentiation foci were never observed, suggesting that alone they do not act as instructive factors for RGC differentiation. Likewise, the candidate repressors KPNA4, MCM2, Idax, RBPMS2, ELG and Zfp161 (Figure 5e, f) down-regulated Ath5 in vivo and inhibited neurogenesis. Three of the candidates tested, NHL-protein, Cbx7 and Islet-2, did not significantly alter reporter expression, although they exhibited a clear effect in the screen and the dose-response analysis (Figure $5 \mathrm{f}$ ). Taken together, $75 \%$ of the candidates tested clearly regulate Ath 5 expression in vivo and activate or repress Ath 5 as predicted from the in vitro assays.

Here, we present a comprehensive TRS with a detailed analysis of candidate expression patterns relative to Ath 5 during the neurogenic wave. We analyze the subcellular localization of previously uncharacterized candidates and show that identified proteins regulate RGC neurogenesis in vivo in the medaka retina. Our data highlight the power of the technology to obtain an enriched set of true-positive regulators from an unbiased collection of full-length cDNAs.

\section{Discussion}

The identification of the components of GRNs is essential to understand how specific developmental programs are executed during embryogenesis [47]. An increasing number of regulatory interactions have been already identified through 


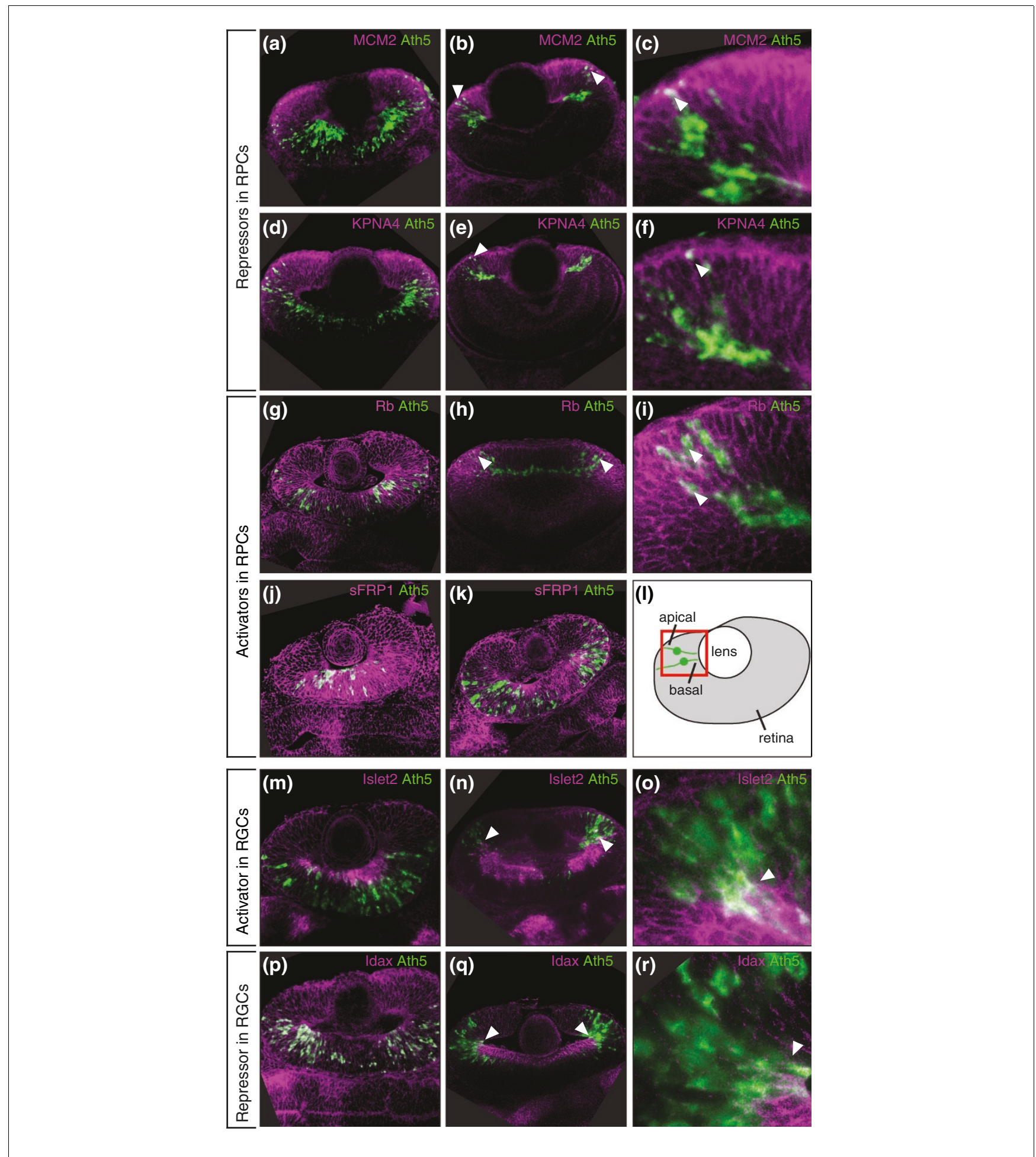

Figure 3

Double-fluorescent whole-mount in situ hybridization of candidates. Ath5 mRNA was detected using TSA-fluorescein (shown in green), and regulator mRNA was visualized using FastRed staining (shown in purple). (a-f) Group I, repressors in RPCs. (g-k) Group 2, activators in RPCs. (I) A schematic representation of a SWS retina. The box demarcates the magnification shown in the close-ups of the transition zone of Ath5 and candidate regulator expression. (m-o) Group 3, activators in RGCs. (p-r) Group 3, repressors in RGCs. In this and subsequent figures, all images are single horizontal confocal sections of the developing eye at the level of the lens, anterior is to the left. Arrowheads point to sites of co-expression of Ath5 and the candidate regulator. 


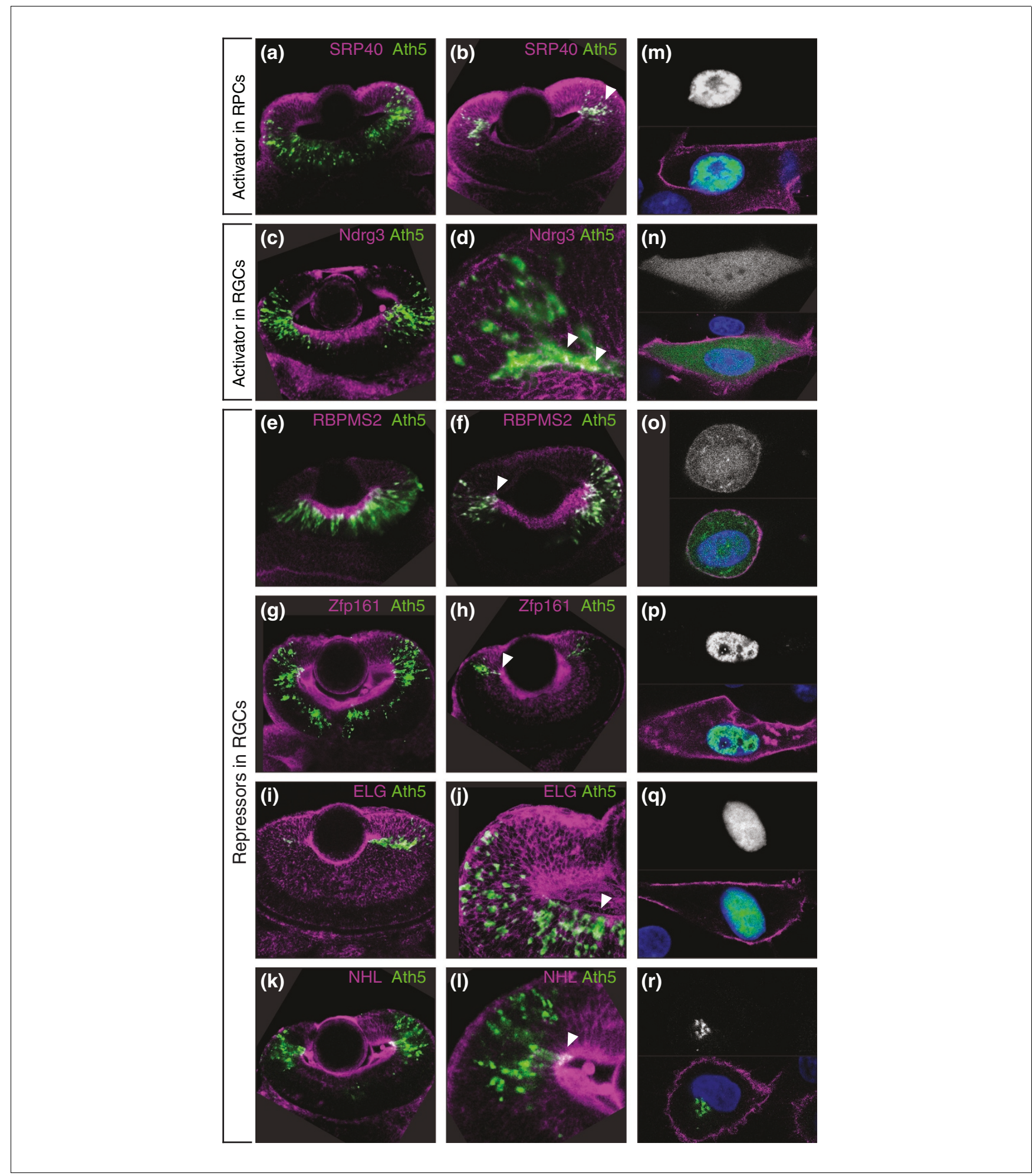

\section{Figure 4}

Double-fluorescent whole-mount in situ hybridization (DFWIS) of novel regulators and subcellular localization. DFWIS A-L. Ath5 mRNA was detected using TSA-fluorescein (green), and regulator mRNA was visualized using FastRed staining (purple). (a, b) Group I, activators in RPCs. (c, d) Group 3, activators in RGCs. (e-I) Group 4, repressors in RGCs. (m-r) Cellular localization. BHK2I cells were transfected with GFP-fusion proteins. The upper half of each image shows the single channel including the GFP-fusion protein. The lower half of each image shows an overlay of the GFP-fusion protein (green), DAPI-stained nucleus (blue) and lynd-Tomato stained cell membrane (purple). 
(a)

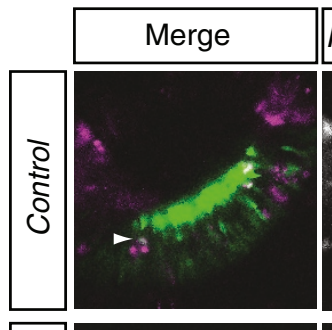

(b)

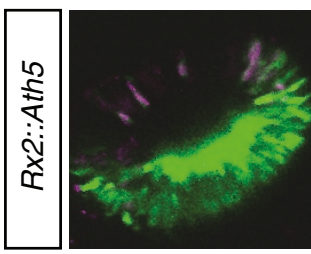

(c)

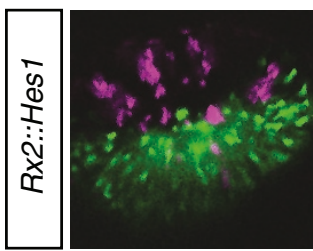

(d)

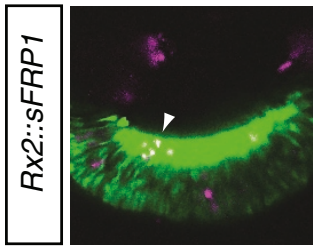

(e)

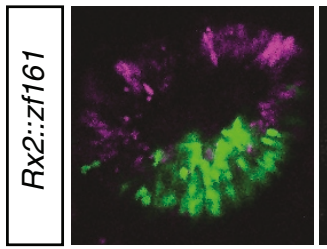

(f)
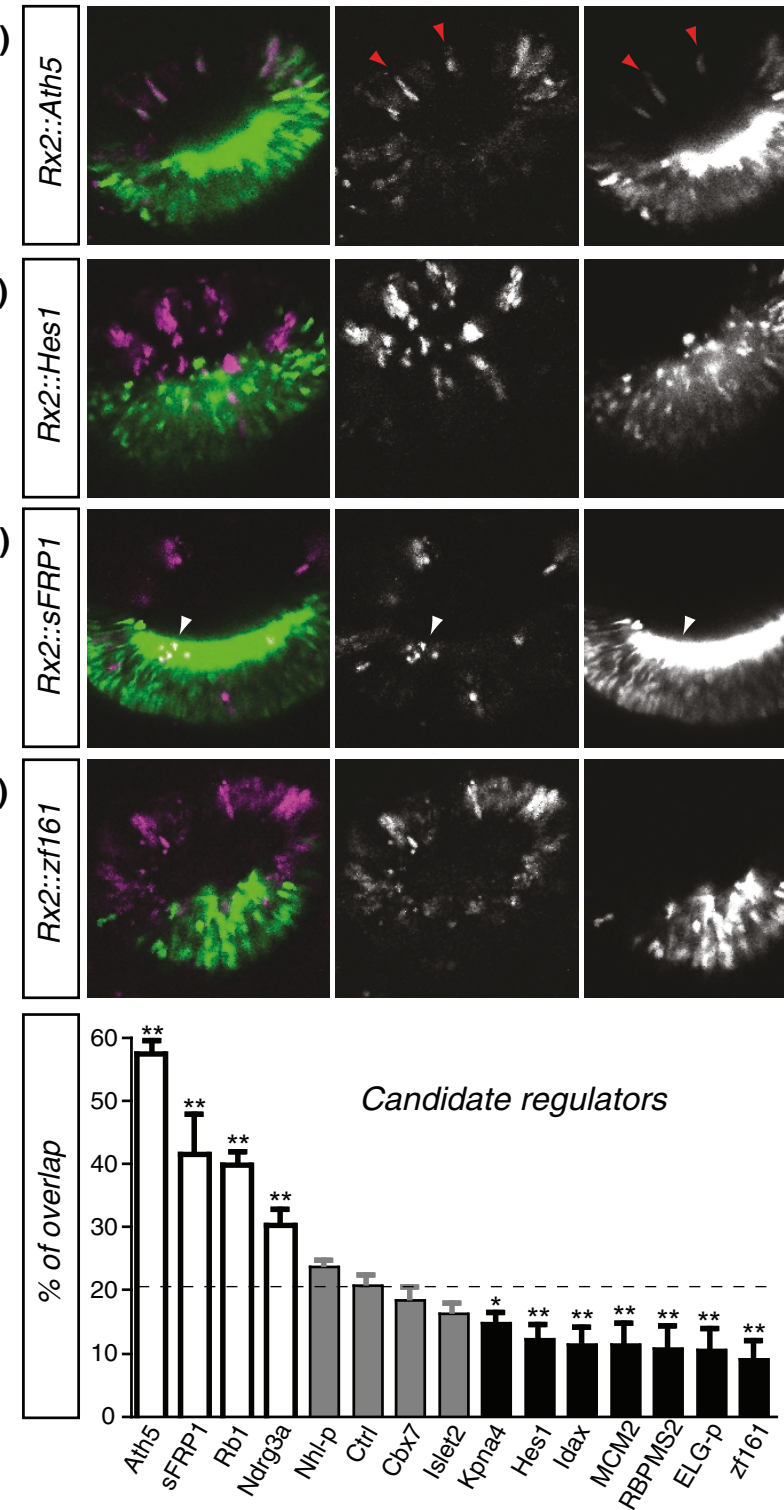
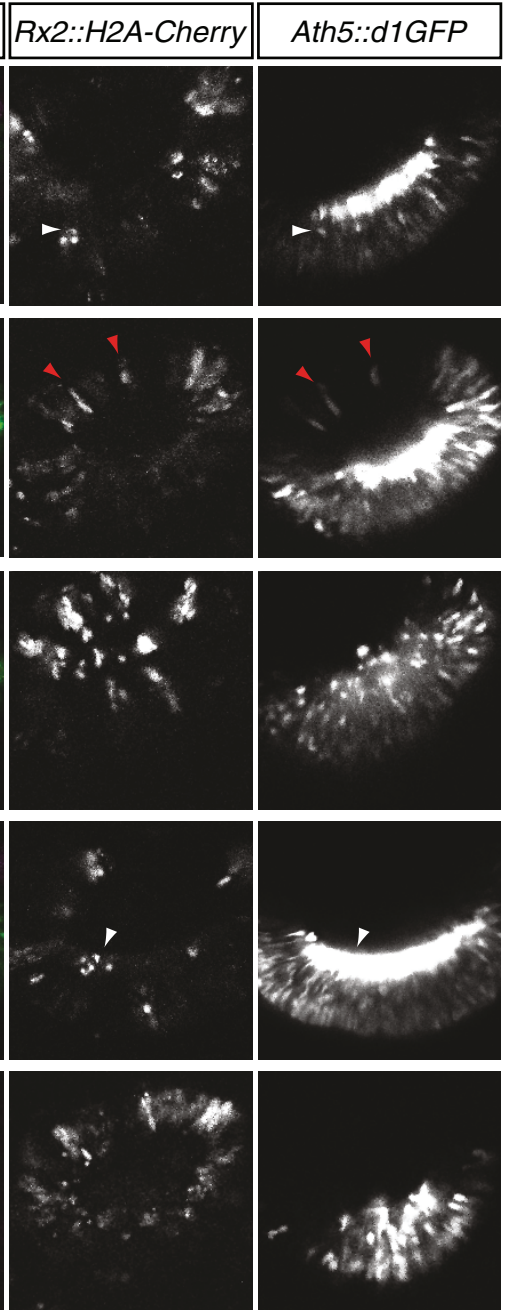

Figure 5

Targeted overexpression analysis. (a-e) Reporter expression. Optical confocal sections through stage 26 retina of Ath5::dIGFP transgenic medaka at the level of the lens. Embryos were co-injected with $R \times 2::$ candidate and $R \times 2::$ nuclearCherry at the one-cell stage. White arrowheads indicate representative double-labeled cells. Red arrowheads indicate the ectopic differentiation of Ath5-positive neurons in the peripheral retina upon Ath5 over-expression (f) Analysis of reporter overlap. For each candidate the percentage of overlap between the regulator and Ath5-positive cells is plotted, with error bars indicating the standard error. The significance of the differences was explored by one-way Anova analysis followed by Dunnett's post-tests to compare each value with the control. Values significantly higher $(P<0.0 \mathrm{I})$ than the control are shown by white bars, and percentages significantly lower by black bars. Percentages that deviate non-significantly from the control are shown by grey bars. 
microarray analysis, chromatin immunoprecipitation-based methodologies and bioinformatics approaches. While these technologies can be applied to systematically detect gene batteries downstream of a given component of the network and are powerful tools to detect individual upstream inputs, they have limited use as genome-wide screening technologies upstream of a node.

We developed the TRS approach to identify upstream factors from a large collection of cDNAs in an unbiased manner. We focused on genes acting in the control of Ath5-mediated RGC specification.

\section{The transregulation screen robustly identifies high- confidence candidate regulators of Ath5}

The TRS approach represents a high-throughput method to explore the transregulatory activity of transcriptome-scale sets of genes acting on native promoter sequences. Using a rapid screening procedure and taking advantage of a highquality medaka unigene expression library, our procedure overcomes many of the limitations of previously used approaches. We benchmarked the assay setup using the known Ath5 regulators Hes-1, Pax6 and Ath5 itself, confirming that Hes1 represses the Ath 5 promoter, while Pax6 and Ath5 activate the promoter under screening conditions. We have screened $44 \%$ of the protein-coding genes predicted in medaka (Ensembl 50) for their activity on the Ath 5 promoter. The internal control provided by a dual luciferase-based approach allows efficient data normalization. Due to the basal activity of the native promoter, both putative repressors and activators are identified in a single screen.

The TRS intrinsically favors the identification of direct regulators whose input on Ath 5 may be mediated either by direct binding to the promoter or through their participation as cofactors in transcriptional complexes. Accordingly, the collection of candidate genes identified is enriched in transcriptional regulators: $44.3 \%$ of candidates are annotated as nucleic acid binding proteins (22\% as transcription factors), while the percentage of transcription factors in vertebrate genomes is estimated at between 5 and 10\% [48]. In fact, most of the previously uncharacterized candidates show nuclear localization. By screening the activity of medaka proteins on a medaka promoter in the context of a mammalian cell line, only strongly conserved interactions were picked up by the screen. Notably, the screen was not restricted to direct regulators only, and also identified a number of upstream regulators, such as SFRP1, a secreted molecule that has been shown to promote RGC differentiation in chicken [23].

To limit the number of selected genes to high-confidence candidates, we applied very stringent selection criteria (Figure 2b), accepting a number of false-negatives. In general, the rate of false negatives obtained by TRS will depend critically on the quality and coverage of the reference cDNA library as well as on the DNA preparations employed during the proce- dure. In our analysis we further discriminated false-positives by employing a pipeline of two nested screens, firstly collecting luciferase data and secondly obtaining spatio-temporal information about the candidate genes in the in situ hybridization screen. Indeed, we identified a number of transcription factors that clearly regulate the promoter but are expressed elsewhere in the embryo. Interestingly, each of these TFs belonged to a protein family out of which at least one member was found to be expressed in the eye. The expression pattern filter efficiently removed these false entries and the number of candidates was thus reduced from 93 to 41 genes with a spatio-temporal expression specific to subdomains of the eye.

To assess the relevance of the findings in the context of an embryo, we carried out functional assays in vivo and found that 9 out of 12 genes tested by clonal analysis in the developing medaka retina regulate Ath5. This in vivo validation demonstrates the power of the approach to efficiently identify high-confidence targets. We classified our candidate regulators into four different groups according to expression pattern and regulatory activity:

\section{Four spatially and functionally distinct sets of regulators define different phases of Ath5 expression Activation phase \\ Group I genes}

Group 1 genes repress the Ath 5 promoter to prevent premature neurogenesis. Genes for MCM2, a member of the general replication initiation complex, and an importin, KPNA4, are expressed with Ath 5 only prior to exit from the cell cycle in the most apical cells and repress the Ath 5 promoter in the context of the retina (Figure 5b), maintaining the RPCs in an undifferentiated state. Interestingly, MCM2 downregulation has been correlated with cell cycle exit and $\mathrm{Rb}$ hyperphosphorylation [49].

\section{Group 2 genes}

Group 2 genes act as initial activators and are expressed in RPCs and nascent RGCs. We demonstrate that the cell cycle exit regulator $\mathrm{Rb}$ activates the Ath5 promoter in vivo. The onset of $\mathrm{Rb}$ overlaps with the last mitosis in apically located RPCs at the onset of Ath 5 expression. A link between cell cycle exit and RGC specification has long been proposed [27] and loss of $\mathrm{Rb}$ has been reported to cause neuronal differentiation defects [50]. Our findings further indicate that the Rb pathway molecularly links cell-cycle exit and activation of the proneural gene Ath5. Interestingly, in Drosophila eye imaginal discs, increased $R b$ expression flanking the atonal domain in the furrow has been reported [51], indicating that part of the atonal/Ath5 gene network is evolutionarily conserved.

We also show that the Wnt-signal modulator sFRP1 activates Ath 5 in vitro and in vivo. This substantiates and extends a previous report showing that $s F R P 1$ favors generation of RGCs and photoreceptors in the developing chicken retina 
[23]. These findings indicate that transregulatory input from both cell-intrinsic factors and extracellular signals converges on the Ath 5 promoter to precisely control the timing of RGC differentiation.

\section{Downregulation phase \\ Group 3 genes}

Group 3 genes maintain Ath 5 expression in basal RGCs just prior to the downregulation of Ath5. Several reports indicate that Ath 5 expression is maintained through an auto-regulatory feedback loop in migrating RGCs [30,33]. We hypothesize that late activators, such as Islet-2 and Ndrg3, cooperate with this feedback loop to maintain high levels of Ath 5 mRNA until the sharp downregulation prior to RGC terminal differentiation. In agreement with this, it has been shown that islet2 is a downstream target of Ath5 in mice [52].

\section{Group 4 genes}

The last step of Ath5 regulation requires sharp downregulation of the promoter. This repression requires both breaking the auto-regulatory feedback loop and directly repressing the regulatory elements of the promoter. We identified eight repressors that are expressed in late RGCs and overlap with Ath5 only in very few basal cells. RBPMS2, an RNA binding protein, and a chaperone-like ELG-protein [53] repress Ath5 in vivo (Figure $5 \mathrm{~b}$ ). We propose that they are required to break the positive Ath 5 feedback loop on the level of RNA regulation. It is likely that the zinc finger protein $\mathrm{Zfp}-161$ represents a more direct transcriptional regulator of Ath5, based on its protein structure, its nuclear localization, and the clear dose-response. We demonstrate that the Wnt-regulator Idax represses Ath 5 in vivo, consistent with the notion that repression of Wnt signaling is required for the progression of neurogenesis [54].

\section{Conclusions}

Our analysis establishes a regulatory framework of RGC neurogenesis. We have identified novel repressors in proliferating RPCs in addition to the previously described initial repressor Hes1 (Figure 6a). We propose that lifting of repression is accompanied by a rapid activation of the promoter through cell-extrinsic (for example, sFRP1) and cell-intrinsic (for example, $\mathrm{Rb}$ ) inputs. The main phase of strong expression has been previously described as being driven by the Ath5 autoregulatory feedback loop and by NeuroM activity. We have now identified other factors that act during this phase and contribute to sustaining the Ath 5 regulatory loop. Importantly, we describe a number of genes involved in the final downregulation of Ath5 (Figure 6b). These include not only transcription factors, but also regulators of RNA metabolism, enzymes and genes of as yet uncharacterized molecular function.

Our nested screening approach has been highly efficient at identifying relevant inputs to Ath5, a central node in the reti- nal neurogenic GRN. The TRS technology extends the current model of Ath 5 regulation and gives novel insights into the mechanisms of retinal neurogenesis.

\section{Materials and methods Medaka stocks}

The Cab-strain of wild-type medaka (Oryzias latipes) were kept in closed stocks at the EMBL, as described [55]. Embryonic stages are according to [56]. For stages after the onset of eye pigmentation the Heino strain was used [57].

\section{Unigene full-length library and reporter vector}

Total RNA was extracted from medaka stages 18, 24, 32 and adults. mRNA was isolated using polyT-beads. The normalized full-length cDNA library was prepared using standard reverse transcription. The cDNAs were cloned into pCMVSport6.1 vector. We sequenced and clustered 55,296 clones based on sequence alignment. One bacterial clone of each cluster was transferred into a consolidated library in a 384well plate by the in-house genomics service. In addition to 13,837 clones from clusters in the initial library, 3,689 clones not sequenced successfully were included.

\section{Reporter vector}

A $3-\mathrm{kb}$ fragment upstream of the medaka Ath5 gene (ENSORLGooooo013722) was amplified using specific primers (forward primer, TGCATCTTCAGCGCAGTGGCA; reverse primer, GGTTTCTGTGCAAAGAGGCGAA) and cloned into the pGL3 luciferase reporter vector that contains the Photinus pyralis luciferase gene.

\section{Cell culture and transfection}

Syrian Hamster Fibroblast (BHK21) cells were cultivated in Dulbecco's modified Eagle's medium (DMEM), supplemented with $10 \%$ fetal calf serum, $200 \mathrm{U} / 1$ penicillin, $200 \mu \mathrm{g} /$ 1 streptomycin and $2 \mathrm{mM}$ L-glutamine. Cells were incubated at $37^{\circ} \mathrm{C}$ in a humidified atmosphere with $5 \% \mathrm{CO}_{2}$. For luciferase assays cells were seeded in white 96-well plates and grown for 5 h. pGL3 Ath5::luc vector (40 ng) and pRL-CMV control vector (5 ng) (Promega, Mannheim, Germany) were incubated with $2 \mu \mathrm{l}$ (10 to $300 \mathrm{ng}$ ) of pCMV-Sport6.1::cDNA in a 3- to 6-fold volume-excess of FuGENE6 transfection reagent in serum-free medium for 30 minutes and then added to the cells. After $42 \mathrm{~h}$ growth at $37^{\circ} \mathrm{C}$, the medium was removed and cells were lysed with $20 \mu$ of $1 \times$ passive lysis buffer (Promega). Each clone was assayed in triplicate.

Dose-response behavior of the candidate regulators was assessed in triplicate by co-transfecting $20,40,80$ or $160 \mathrm{ng}$ of pCMV-Sport6.1::cDNA with the reporter and control construct. The total amount of DNA transfected in each doseresponse experiment was kept constant by adding pCS2+ vector where appropriate (see supplementary methods in Additional data file 1 ). 
(a) Rapid activation of Ath5

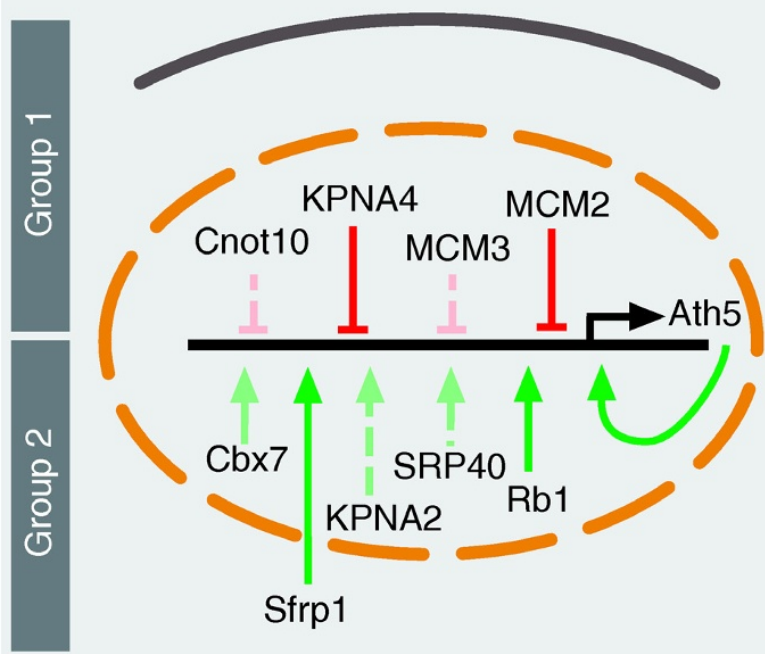

(b) Downregulation of Ath5

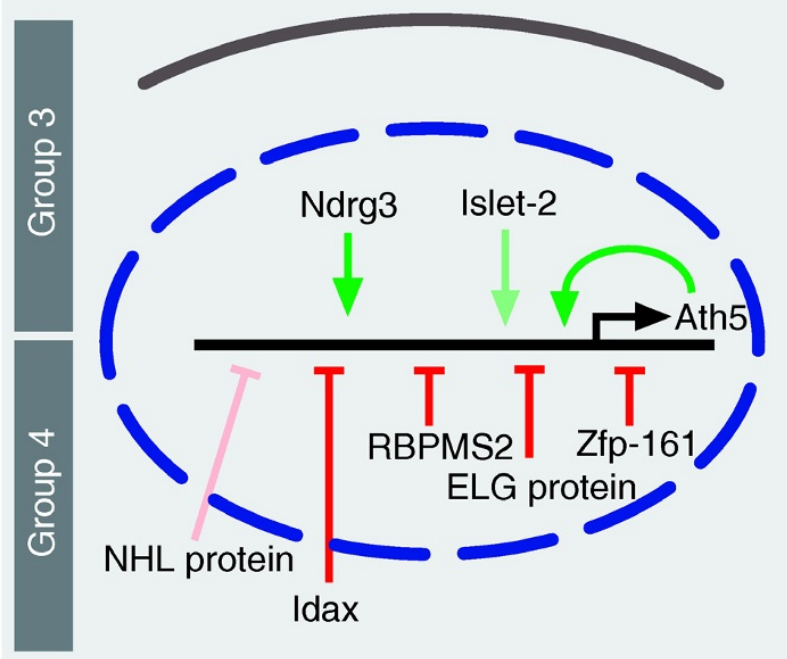

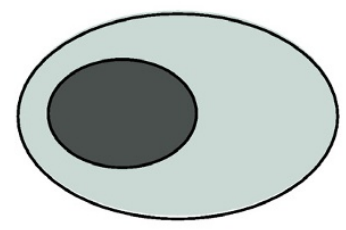

proliferating RPC

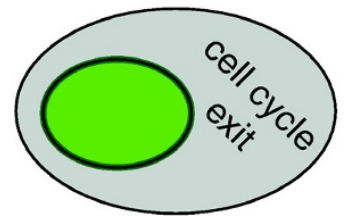

Proneural

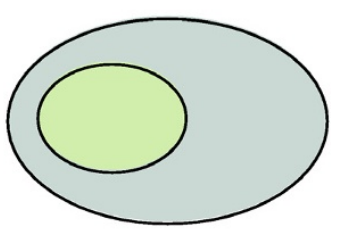

differentiating RGC

\section{Figure 6}

Model of Ath5 expression dynamics. Summary of input on the 3-kb Ath5 promoter fragment. Solid lines represent activities that were confirmed in vivo, and shaded solid lines represent regulatory activities that were not significant in vivo. (a) Onset of Ath5 expression. Group I genes such as MCM2 and KPNA4 may act to prevent premature Ath5 expression. Ath5 expression is switched on by group 2 genes, such as sFRPI and Rb. (b) Downregulation of Ath5. Group 3 genes: Ndrg3 and Islet-2 may prevent premature downregulation of Ath5. Expression of Ath5 is downregulated by signals through Idax and the regulatory activity of RBPMS2, ELG-protein and Zfp-16I (group 4).

\section{Luciferase assays}

Raw luminescence values were sequentially recorded in a Victor Light Luminescence counter (PerkinElmer Waltham, Massachusetts, USA) using a dual flash luciferase system (Promega) according to manufacturer's guidelines. All values were stored in a FileMaker database (FileMaker, Inc, Santa Clara, California, USA) The median of the raw ratio between the firefly luciferase and the Renilla luciferase of each triplicate was normalized against the median of all ratios of the 96well plates containing the triplicates. Clones with a non-random deviation from the average were selected as candidate regulators (see supplementary methods in Additional data file 1). When only one assay was available for a clone, no standard deviation filter was applied.

\section{Riboprobe preparation}

The insert with a 3 ' flanking T7-promoter was amplified from the pCMV-Sport6.1 backbone using standard PCR with M13frw and M13rev primers. T7 RNA polymerase-based transcription was performed as previously described [58].

\section{Robotic-assisted whole-mount in situ hybridization}

Fixation, protein $\mathrm{K}$ digestion and post-fixation of embryos were carried out as previously described [58]. Solutions were prepared according to standard protocols, except phosphatebuffered saline-Tween 20 (PTW) for the washes containing $0.35 \%$ PTW. In addition to the standard protocol, a pre-staining buffer (o.1 M Tris pH7.5, $25 \mathrm{mM} \mathrm{NaCl,} \mathrm{0.1 \%} \mathrm{Tween20)}$ was prepared. Hybridization and washes were carried out in an in situ robot (InSitu Pro, Intavis Koeln, Germany) with an 
adapted in situ protocol (see supplementary Materials and methods in Additional data file 1). The staining reactions were performed manually as previously described [58].

\section{Double-fluorescence in situ hybridization}

Embryos were prepared as described above for single-color in situ hybridization. Fluorescein-labeled probes were generated against Ath5 according to standard protocol [59]. Embryos were hybridized with a mixture of this probe and a DIG-labeled probe against the candidate regulator. Anti-fluorescein antibody ( $\alpha$-Fluo Ab) coupled to peroxidase and antidigoxigenin antibody ( $\alpha$-Dig Ab) coupled to alkaline phosphatase were mixed in blocking buffer at 1:500 and 1:1,00o dilutions, respectively. Embryos were first incubated with TSA-fluorescein according to the manufacturer's instructions (PerkinElmer) for 60 minutes at room temperature in the dark. The DIG-probes were visualized with FastRed staining (Roche Mannheim, Germany).

\section{Imaging}

Images from embryos mounted in toto were acquired on a Leica SP2 confocal microscope using a HCX PL APO CS 40×, 1.25 oil objective. Images were assembled and processed in Adobe Photoshop and ImageJ. Brightness and contrast were adjusted; filtering was applied for noise reduction and thresholding when needed.

\section{Conservation analysis and transcription factor binding site discovery}

Conserved sequences in the 3 -kb upstream sequence of Ath 5 were determined as previously described [31]. Position weight matrices for the TFBSs were obtained from TRANSFAC [44] (NFkB Identifier: Moo19); for islet-2 we used the position weight matrix for the LIM- and homeodomain-containing transcription factor Lhx3 (Mo0510). Potential TFBSs were search for using Possum [6o], using a threshold of 4 and a relative abundance range of 50 for Moo194 and 100 for Moo510.

\section{Fusion protein and localization assays}

The proteins were amplified from pCMV-Sport6.1 using the specific primers (see supplementary Materials and methods in Additional data file 1). Forward primers contained restriction sites creating an EcoRI compatible overhang and the reverse primers a restriction site creating an NcoI compatible overhang. The sequence verified fragments were subcloned into a pCS2+ hGFP vector.

BHK21 cells were grown on human fibronectin-coated culture slides to 40 to $50 \%$ confluency and transfected with $0.8 \mu \mathrm{g}$ of each fusion protein and $0.2 \mu \mathrm{g}$ of pCS2+ lynd-tomato for membrane labeling. After $42 \mathrm{~h}$ cells were fixed in $4 \%$ paraformaldehyde/phosphate-buffered saline, permeabilized in 0.2\%Triton/phosphate-buffered saline and stained with DAPI. The slides were sealed with a glass coverslip and were kept at $4^{\circ} \mathrm{C}$ in the dark.

\section{Functional assays}

Candidate regulators in pCMV-Sport6.1 were cloned into a gateway recombination destination vector containing a 2.4kb OlRx2 promoter fragment driving expression in the eye [61]. Each construct $(5 \mathrm{ng} / \mu \mathrm{l})$ was individually co-injected with Rx2::H2A-Cherry $(7.5 \mathrm{ng} / \mu \mathrm{l})$ into one-cell stage medaka embryos as previously described [46]. Stage 26 embryos were protein K treated to remove hairs from the chorion, heptanol treated $(3 \mathrm{mM})$ and imaged in vivo using a Leica SPE confocal microscope with a $40 \times$ dipping lens. Retina with clonal expression of the constructs were imaged individually at the level of the lens. H2A-Cherry-expressing cells within the Ath5-expression domain were scored for Ath5 coexpression.

\section{Statistical analysis}

Quantitative data are expressed as mean \pm standard error of the mean. Significant differences among groups were evaluated by one-way ANOVA followed by Dunnett's tests (GraphPad Prism. GraphPad Software, Inc. San Diego, California, USA) and are indicated when relevant.

\section{Abbreviations}

bHLH: basic helix-loop-helix; GFP: green fluorescent protein; GRN: gene regulatory network; IS: initiation stage; PS: progression stage; Rb: Retinoblastoma; RGC: retinal ganglion cell; RPC: retinal progenitor cell; sFRP: secreted frizzled related protein; SWS: steady wave stage; TFBS: transcription factor binding site; TRS: trans-regulation screen.

\section{Authors' contributions}

JRMM and JW conceived the study. JRMM and MS designed, coordinated and carried out the screening pipeline. MS designed and maintained the screen database. BW carried out the semi-automated in situ hybridizations. PM participated in the double fluorescence whole-mount in situ hybridization experiments. The manuscript was initially drafted by MS. JRMM and JW worked on further versions of the text. All authors read and approved the final manuscript.

\section{Additional data files}

The following additional data are available with the online version of this paper: a PDF including supplementary Tables S1 and Table S2, supplementary Figures S1 to S4, and supplementary Materials and methods (Additional data file 1).

\section{Acknowledgements}

We are grateful to Katherine Brown and Lazaro Centanin for their critical input and discussion, the GeneCore Facility, EMBL, for re-arraying the cDNA library and making 96-well format minipreps, A Nowicka and D Hofmann for fish husbandry, and C Müller for technical assistance. JRMM was supported by EMBO and Marie Curie fellowships and by the Ramon y Cajal program. This work was supported by grants from the Deutsche Forschungsgemeinschaft, Collaborative Research Centre 488, the EU to JW. 


\section{References}

I. Erwin D, Davidson E: The evolution of hierarchical gene regulatory networks. Nat Rev Genet 2009, 10:|4|-| 48.

2. Mu X, Fu X, Sun H, Beremand PD, Thomas TL, Klein WH: A gene network downstream of transcription factor Math5 regulates retinal progenitor cell competence and ganglion cell fate. Dev Biol 2005, 280:467-48।

3. Cremisi F, Philpott A, Ohnuma S: Cell cycle and cell fate interactions in neural development. Curr Opin Neurobiol 2003, 13:26-33.

4. Livesey FJ, Cepko CL: Vertebrate neural cell-fate determination: lessons from the retina. Nat Rev Neurosci 2001, 2: 109-I I8.

5. Marquardt T, Gruss P: Generating neuronal diversity in the retina: one for nearly all. Trends Neurosci 2002, 25:32-38.

6. Ohnuma S, Harris WA: Neurogenesis and the cell cycle. Neuron 2003, 40:199-208.

7. Del Bene F, Wehman AM, Link BA, Baier H: Regulation of neurogenesis by interkinetic nuclear migration through an apicalbasal notch gradient. Cell 2008, I 34: I055-1065.

8. Frade JM: Interkinetic nuclear movement in the vertebrate neuroepithelium: encounters with an old acquaintance. Prog Brain Res 2002, I36:67-71.

9. Sauer FC: The interkinetic migration of embryonic epithelial nuclei. J Morphol 1936, 60:I-II.

10. Brown NL, Patel S, Brzezinski J, Glaser T: Math5 is required for retinal ganglion cell and optic nerve formation. Development 200I, I 28:2497-2508.

II. Kay JN, Finger-Baier KC, Roeser T, Staub W, Baier H: Retinal ganglion cell genesis requires lakritz, a Zebrafish atonal homolog. Neuron 200I, 30:725-736.

12. Wang SW, Kim BS, Ding K, Wang H, Sun D, Johnson RL, Klein WH, Gan L: Requirement for math 5 in the development of retinal ganglion cells. Genes Dev 200 I, I 5:24-29.

13. Kanekar S, Perron M, Dorsky R, Harris WA, Jan LY, Jan YN, Vetter ML: Xath5 participates in a network of bHLH genes in the developing Xenopus retina. Neuron 1997, 19:981-994.

14. Liu W, Mo Z, Xiang M: The Ath5 proneural genes function upstream of Brn3 POU domain transcription factor genes to promote retinal ganglion cell development. Proc Natl Acad Sci USA 200I, 98: I649-I654.

15. Masai I, Yamaguchi M, Tonou-Fujimori N, Komori A, Okamoto H: The hedgehog-PKA pathway regulates two distinct steps of the differentiation of retinal ganglion cells: the cell-cycle exit of retinoblasts and their neuronal maturation. Development 2005, I 32:1539-1553.

16. Poggi L, Vitorino M, Masai I, Harris WA: Influences on neural lineage and mode of division in the zebrafish retina in vivo. J Cell Biol 2005, I 71:991-999.

17. Bonini NM, Choi KW: Early decisions in Drosophila eye morphogenesis. Curr Opin Genet Dev 1995, 5:507-5I5.

18. Hu M, Easter SS: Retinal neurogenesis: the formation of the initial central patch of postmitotic cells. Dev Biol 1999, 207:309-321.

19. Masai I, Stemple DL, Okamoto H, Wilson SW: Midline signals regulate retinal neurogenesis in zebrafish. Neuron 2000, 27:25I-263.

20. Laessing U, Stuermer CA: Spatiotemporal pattern of retinal ganglion cell differentiation revealed by the expression of neurolin in embryonic zebrafish. J Neurobiol 1996, 29:65-74.

21. Prada C, Puga J, Perez-Mendez L, Lopez R, Ramirez G: Spatial and temporal patterns of neurogenesis in the chick retina. Eur J Neurosci 199I, 3:559-569.

22. Young RW: Cell differentiation in the retina of the mouse. Anat Rec 1985, 2 I 2: 199-205.

23. Esteve P, Trousse F, Rodriguez J, Bovolenta P: SFRP I modulates retina cell differentiation through a beta-catenin-independent mechanism. J Cell Sci 2003, I I 6:247I-248I.

24. Martinez-Morales JR, Del Bene F, Nica G, Hammerschmidt M, Bovolenta $\mathrm{P}$, Wittbrodt J: Differentiation of the vertebrate retina is coordinated by an FGF signaling center. Dev Cell 2005, 8:565-574.

25. Shkumatava A, Fischer S, Muller F, Strahle U, Neumann CJ: Sonic hedgehog, secreted by amacrine cells, acts as a short-range signal to direct differentiation and lamination in the zebrafish retina. Development 2004, I 3 I:3849-3858.

26. Vinothkumar S, Rastegar S, Takamiya M, Ertzer R, Strahle U: Sequential and cooperative action of Fgfs and Shh in the zebrafish retina. Dev Biol 2008, 3 | 4:200-2 4 .
27. Kay JN, Link BA, Baier $\mathrm{H}$ : Staggered cell-intrinsic timing of ath5 expression underlies the wave of ganglion cell neurogenesis in the zebrafish retina. Development 2005, I 32:2573-2585.

28. Hatakeyama J, Bessho $Y$, Katoh K, Ookawara S, Fujioka M, Guillemot $F$, Kageyama R: Hes genes regulate size, shape and histogenesis of the nervous system by control of the timing of neural stem cell differentiation. Development 2004, I 3 |:5539-5550.

29. Tomita K, Ishibashi M, Nakahara K, Ang SL, Nakanishi S, Guillemot F, Kageyama R: Mammalian hairy and Enhancer of split homolog I regulates differentiation of retinal neurons and is essential for eye morphogenesis. Neuron 1996, 16:723-734.

30. Matter-Sadzinski L, Puzianowska-Kuznicka M, Hernandez J, Ballivet M, Matter JM: A bHLH transcriptional network regulating the specification of retinal ganglion cells. Development 2005, I32:3907-3921.

31. Del Bene F, Ettwiller L, Skowronska-Krawczyk D, Baier H, Matter JM, Birney $\mathrm{E}$, Wittbrodt J: In vivo validation of a computationally predicted conserved Ath5 target gene set. PLoS Genet 2007, 3:|66|-|67|

32. Skowronska-Krawczyk D, Ballivet M, Dynlacht BD, Matter JM: Highly specific interactions between bHLH transcription factors and chromatin during retina development. Development 2004, I 3 I:4447-4454.

33. Hernandez J, Matter-Sadzinski L, Skowronska-Krawczyk D, Chiodini F, Alliod C, Ballivet M, Matter JM: Highly conserved sequences mediate the dynamic interplay of basic helix-loop-helix proteins regulating retinogenesis. J Biol Chem 2007, 282:37894-37905.

34. Hufnagel RB, Riesenberg AN, Saul SM, Brown NL: Conserved regulation of Math5 and Math I revealed by Math5-GFP transgenes. Mol Cell Neurosci 2007, 36:435-448.

35. Riesenberg AN, Le TT, Willardsen MI, Blackburn DC, Vetter ML, Brown NL: Pax6 regulation of Math5 during mouse retinal neurogenesis. Genesis 2009, 47: $175-187$.

36. Willardsen MI, Suli A, Pan Y, Marsh-Armstrong N, Chien CB, ElHodiri H, Brown NL, Moore KB, Vetter ML: Temporal regulation of Ath5 gene expression during eye development. Dev Biol 2009, 326:47|-48I.

37. Deplancke B, Dupuy D, Vidal M, Walhout AJ: A gateway-compatible yeast one-hybrid system. Genome Res 2004, I 4:2093-2 I0I.

38. Berger MF, Badis G, Gehrke AR, Talukder S, Philippakis AA, PenaCastillo L, Alleyne TM, Mnaimneh S, Botvinnik OB, Chan ET, Khalid F, Zhang W, Newburger D, Jaeger SA, Morris QD, Bulyk ML, Hughes TR: Variation in homeodomain DNA binding revealed by high-resolution analysis of sequence preferences. Cell 2008 , I33:1266-1276.

39. Noyes MB, Christensen RG, Wakabayashi A, Stormo GD, Brodsky $\mathrm{MH}$, Wolfe SA: Analysis of homeodomain specificities allows the family-wide prediction of preferred recognition sites. Cell 2008, I33:1277-1289.

40. Liu Y, Kern JT, Walker JR, Johnson JA, Schultz PG, Luesch H: A genomic screen for activators of the antioxidant response element. Proc Natl Acad Sci USA 2007, 104:5205-5210.

4I. Fan F, Wood KV: Bioluminescent assays for high-throughput screening. Assay Drug Dev Technol 2007, 5:127-136.

42. Boutros M, Brás LP, Huber W: Analysis of cell-based RNAi screens. Genome Biol 2006, 7:R66.

43. Medaka Expression Pattern Database [http://ani.embl.de:8080/ mepd/]

44. Matys V, Fricke E, Geffers R, Gossling E, Haubrock M, Hehl R, Hornischer K, Karas D, Kel AE, Kel-Margoulis OV, Kloos DU, Land S, Lewicki-Potapov B, Michael H, Münch R, Reuter I, Rotert S, Saxel H, Scheer $M$, Thiele S, Wingender E: TRANSFAC: transcriptional regulation, from patterns to profiles. Nucleic Acids Res 2003, 3 1:374-378.

45. Okuda T, Kokame K, Miyata T: Differential expression patterns of NDRG family proteins in the central nervous system. J Histochem Cytochem 2008, 56: 175-182.

46. Rembold M, Lahiri K, Foulkes NS, Wittbrodt J: Transgenesis in fish: efficient selection of transgenic fish by co-injection with a fluorescent reporter construct. Nat Protoc 2006, I: I I33- I I 39.

47. Stathopoulos A, Levine M: Genomic regulatory networks and animal development. Dev Cell 2005, 9:449-462.

48. Levine $M$, Tjian $R$ : Transcription regulation and animal diversity. Nature 2003, 424:|47-|5|.

49. Noseda M, Niessen K, McLean G, Chang L, Karsan A: Notchdependent cell cycle arrest is associated with downregulation of minichromosome maintenance proteins. Circ Res 2005 , 
97:102-104.

50. Chen D, Opavsky R, Pacal M, Tanimoto N, Wenzel P, Seeliger MW, Leone G, Bremner R: Rb-mediated neuronal differentiation through cell-cycle-independent regulation of E2f3a. PLoS Biol 2007, 5: el79.

5I. Keller SA, Ullah Z, Buckley MS, Henry RW, Arnosti DN: Distinct developmental expression of Drosophila retinoblastoma factors. Gene Expr Patterns 2005, 5:4III-42I.

52. Yang Z, Ding K, Pan L, Deng M, Gan L: Math5 determines the competence state of retinal ganglion cell progenitors. Dev Biol 2003, 264:240-254.

53. Merz C, Urlaub H, Will CL, Luhrmann R: Protein composition of human $m R N P s$ spliced in vitro and differential requirements for mRNP protein recruitment. RNA 2007, 13:1 | $6-128$.

54. Yamaguchi M, Tonou-Fujimori N, Komori A, Maeda R, Nojima Y, Li $\mathrm{H}$, Okamoto H, Masai I: Histone deacetylase I regulates retinal neurogenesis in zebrafish by suppressing Wnt and Notch signaling pathways. Development 2005, I 32:3027-3043.

55. Koster R, Stick R, Loosli F, Wittbrodt J: Medaka spalt acts as a target gene of hedgehog signaling. Development 1997, |24:3|47-3|56.

56. Iwamatsu T: Stages of normal development in the medaka Oryzias latipes. Mech Dev 2004, I 21:605-6I8.

57. Loosli F, Koster RW, Carl M, Kuhnlein R, Henrich T, Mucke M, Krone A, Wittbrodt J: A genetic screen for mutations affecting embryonic development in medaka fish (Oryzias latipes). Mech Dev 2000, 97:133-139.

58. Loosli F, Winkler S, Burgtorf C, Wurmbach E, Ansorge W, Henrich T, Grabher C, Arendt D, Carl M, Krone A, Grzebisz E, Wittbrodt J: Medaka eyeless is the key factor linking retinal determination and eye growth. Development 200I, I 28:4035-4044.

59. Loosli F, Koster RW, Carl M, Krone A, Wittbrodt J: Six3, a medaka homologue of the Drosophila homeobox gene sine oculis is expressed in the anterior embryonic shield and the developing eye. Mech Dev 1998, 74:159-164.

60. Possum web page [http://zlab.bu.edu/ mfrith/possum/]

61. Martinez-Morales JR, Rembold M, Greger K, Simpson JC, Brown KE, Quiring R, Pepperkok R, Martin-Bermudo MD, Himmelbauer H, Wittbrodt J: ojoplano-mediated basal constriction is essential for optic cup morphogenesis. Development 2009, 136:2।65-2I75. 